\title{
In vivo Imaging and Drug Storage by Quantum-Dot-Conjugated Carbon Nanotubes**
}

\author{
By Yan Guo, Donglu Shi, ${ }^{*}$ Hoonsung Cho, Zhongyun Dong, Amit Kulkarni, Giovanni M. Pauletti, Wei Wang, \\ Jie Lian, Wen Liu, Lei Ren, Qiqing Zhang, Guokui Liu, Christopher Huth, Lumin Wang, and Rodney C. Ewing
}

A specially designed carbon nanotube (CNT) is developed for use in the early detection and treatment of cancer. The key functionalities for biomedical diagnosis and drug delivery are incorporated into the CNTs. In vivo imaging of live mice is achieved by intravenously injecting quantum dot (QD)-conjugated CNT for the first time. With near infrared emission around $752 \mathrm{~nm}$, the CNT with surface-conjugated QD (CNT-QD) exhibit a strong luminescence for non-invasive optical in vivo imaging. CNT surface modification is achieved by a plasma polymerization approach that deposited ultra-thin acrylic acid or poly(lactic$c o$-glycolic acid) (PLGA) films $(\sim 3 \mathrm{~nm})$ onto the nanotubes. The anticancer agent paclitaxel is loaded at $112.5 \pm 5.8 \mu \mathrm{g} \mathrm{mg}^{-1}$ to PLGA-coated CNT. Cytotoxicity of this novel drug delivery system is evaluated in vitro using PC-3MM2 human prostate carcinoma cells and quantified by the 3-(4,5-dimethylthiazol-2-yl)-2,5-diphenyltetrazolium bromide (MTT) assay. The in vivo distribution determined by inductively coupled plasma mass spectrometry (ICP-MS) indicates CNT-QD uptake in various organs of live animals.

[*] Prof. D. Shi, Y. Guo, H. Cho, W. Wang, C. Huth Department of Chemical and Materials Engineering University of Cincinnati

Cincinnati, OH 45221 (USA)

E-mail: shid@email.uc.edu

Prof. D. Shi

The Research Institute of Micro/Nano Science \& Technology

Shanghai Jiao Tong University and

College of Materials Science and Engineering

Tongji University

Shanghai, 200092 (PR China)

Prof. Z. Dong

Department of Internal Medicine, College of Medicine

University of Cincinnati

Cincinnati, OH 45267 (USA)

Prof. G. M. Pauletti, A. Kulkarni

James L. Winkle College of Pharmacy

University of Cincinnati

Cincinnati, OH 45221 (USA)

Prof. J. Lian

Department of Mechanical, Aerospace and Nuclear Engineering Rensselaer Polytechnic Institute

Troy, NY 12180 (USA)

Prof. L. Wang, Prof. R. C. Ewing

Departments of Geological Sciences, Nuclear Engineering \&

Radiological Sciences and Materials Science \& Engineering

University of Michigan

Ann Arbor, MI 48109 (USA)

W. Liu, Prof. L. Ren, Prof. Q. Zhang

Research Center for Biomedical Engineering, Medical College

Xiamen University

Xiamen, Fujian 361005 (PR China)

Dr. G. Liu

Chemistry Division

Argonne National Laboratory

Argonne, IL 60439 (USA)

\section{Introduction}

One of the key challenges of nanotechnology in cancer diagnosis and treatment has been the design and development of a nanosurface structure with multiple functionalities. Due to complexity of biological systems, these nanostructured materials must have key features, such as the ability to "tune" or control the surface properties. The first step is to treat the surface with specific functional groups such that they attach to specific biological molecules. Second, for diagnosis, the nanoparticle should have a strong luminescence in the visible range for in vivo imaging. Third, certain nanoparticle should have a geometry that allows for storage and release of treatment drugs. Finally, the nanoparticle should be biodegradable in order to avoid toxic effects. Thus far, there have been few attempts to design and produce a nanostructure that meets all of these requirements.

There are several critical issues involved in the design and the development of such a nanoscale material. The first is the surface functionalization by a variety of novel techniques. As reported previously, the physical and chemical properties of CNT can be significantly altered through chemical surface modification, doping, and coating. ${ }^{[1]}$ The surface

[**] The work at University of Cincinnati (UC) was supported by a grant from UC Institute for Nanoscale Science and Technology. The work at Argonne National Laboratory was performed under the auspices of the Office of Basic Energy Science, Division of Chemical Sciences, U.S. Department of Energy, under Contract No. W-31-109-ENG-38. The TEM analyses were conducted at the Electron Microbeam Analysis Laboratory at the University of Michigan and supported by an NSF NIRT grant (EAR-0403732). 
functionalization of CNT by a variety of organic and inorganic species leads to the assembly of novel, hybrid nanomaterials. Such an integrated nanosystem normally requires covalent bonding of the CNT to another nanospecies in a predictable manner. Recent attempts have attached gold to $\mathrm{CNT}^{[2]}$ and $\mathrm{TiO}_{2}$ nanoparticles. ${ }^{[3]}$ However, these methods resulted in undesirable side reactions, producing large clusters of nanoparticles, which were unsuitable for biomedical applications. We have previously described a unique plasma polymerization process that can be used to treat the surfaces of CNT. Ultrathin polymer films $(\sim 3 \mathrm{~nm})$ with functional groups were uniformly deposited on the inner and outer surfaces of CNT. ${ }^{[4-7]}$

For biomedical diagnosis applications, one of the important issues involves the intensity and the frequency of the emissions from the nanoparticles. In particular, for deep tissue tumor imaging, highly intense emissions are required at frequency close to the near infrared range $(>700 \mathrm{~nm})$. Carbon nanotubes (CNTs) are intrinsically fluorescent in the region of near infrared, ${ }^{[8-10]}$ whereas human tissues and biological fluids ${ }^{[11]}$ are practically transparent to these emissions. ${ }^{[12-15]}$ However, the emission intensities are quite low and not useful for deep tissue or live animal imaging. In our previous research we have succeeded in synthesizing CNT with surface-coated luminescent rare-earth materials, but their emissions have low efficiency for in vivo imaging. ${ }^{[16]}$ In contrast, quantum dots (QD), which are semiconducting nanocrystals, may be "tuned" based on their size and have superb optical properties, resulting from quantum confinement effects. ${ }^{[17,18]}$ They offer high resistance to photobleaching, thus making them attractive materials for optoelectronics ${ }^{[19,20]}$ and in vivo biosensing applications. $^{[21]}$ Bruchez et al. ${ }^{[22]}$ and Chan and Nie ${ }^{[23]}$ first reported the use of QD conjugates for labeling biological specimens. Subsequently, several other studies demonstrated the labeling of whole cells and tissue sections using different surface modifications of QD. ${ }^{[24-26]}$ For both diagnosis and treatment, the key issue here is how to combine the tubular structure of CNT and the tunable optical properties of QD in an intravenously (i.v.) deliverable nanoassembly.

Another issue deals with suitable requirements for in vivo imaging. Particularly, QD must have an adequate circulating lifetime, must show minimal non-specific deposition, and must retain their fluorescence for a sufficiently long period. Reduced body clearance can be achieved by coating nanoparticles with hydrophilic polymers such as polyethylene glycol (PEG). This augments vascular circulation time without completely eliminating non-specific tissue uptake. ${ }^{[27-29]}$ Toxicity and biocompatibility have been major concerns for the use of nanomaterials in biomedical applications. ${ }^{[30,31]}$ Before clinical applications of QD become possible, the biocompatibility of these nanoparticles must be thoroughly investigated. For cell culture studies, a biocompatible particle must be non-toxic and inert, as well as stable over the course of an assay. Recently, Derfus et al. ${ }^{[32]}$ used cultured liver cells to determine the cytotoxicity of $\mathrm{CdSe} /$ $\mathrm{ZnS}$ QD with various surface coatings. The results suggested that the surface coatings must be sufficiently stable in order to prevent oxidation of the QD surfaces, which results in the release of divalent cadmium, a known toxin and suspected carcinogen. PEG and other biologically inert polymers may be useful for reducing QD-associated toxicity. So far, nearly all studies on the in vivo use of QD have reported normal organism development and no short-term detectable toxicity. ${ }^{[33-38]}$

Paclitaxel is an antitumor agent demonstrating significant activity in clinical trials against a variety of solid tumors. ${ }^{[39,40]}$ However, limited aqueous solubility and low therapeutic index restrict widespread clinical application. Therefore, a diverse array of materials has been investigated for paclitaxel delivery systems including liposomes, ${ }^{[41]}$ nanoparticles, ${ }^{[42]}$ microspheres, ${ }^{[43]}$ and soluble polymers. ${ }^{[44]}$ Among these new drug delivery systems, polymers have attracted attention as promising carriers for anticancer agents because of increased stability and opportunities of surface functionalization. Among those polymers investigated poly(lactic-co-glycolic acid) (PLGA), which is biodegradable and FDA approved, is preferentially used in drug delivery systems.

In this study, nanotubes with different properties and functionalities were assembled based on a unique nanoscale design. An idealized representation of the nanostructure design for in vivo imaging and drug storage is schematically illustrated in Figure 1. The hollow core and polymer-coated surfaces of the nanotube can be used to store antitumor agents such as paclitaxel as a consequence of non-covalent adsorption. For deep tissue imaging, the outer surface of the nanotube is conjugated with luminescent materials such as QD. However, both drug storage and surface conjugation of QD depicted in Figure 1 present great challenge to the surface functionalization of the CNTs.

In this paper, we report a novel design of a nanoassembly. Using a unique plasma polymerization method, CNT surfaces were functionalized with carboxyl groups that facilitated covalent coupling of amine-containing QD. Successful in vivo imaging and quantitative determination of paclitaxel drug loading are described for the first time for this novel nanoassembly. CNTs containing an ultra-thin PLGA coating were prepared by plasma polymerization. Paclitaxel loading efficiency on PLGA-coated CNTs was measured by high performance liquid chromatography (HPLC). The therapeutic efficacy of paclitaxel-loaded CNT was estimated in vitro by quantifying drug-induced changes in viability of human PC$3 \mathrm{MM} 2$ prostate carcinoma cells using the MTT assay.

\section{Results and Discussion}

\subsection{Plasma Surface Functionalization}

Plasma polymerization is an effective method for surface functionalization of nanotubes and nanoparticles. ${ }^{[4-7]}$ The main principle of plasma polymerization is that the ionized and excited monomer molecules created by an electrical field bombard and react on the surface of the substrate. These activated molecules may be etched, sputtered, or deposited onto the substrate surface. Due to these characteristics, the plasma technique can be used for polymer surface functionalization on various nanotubes and nanoparticles. 


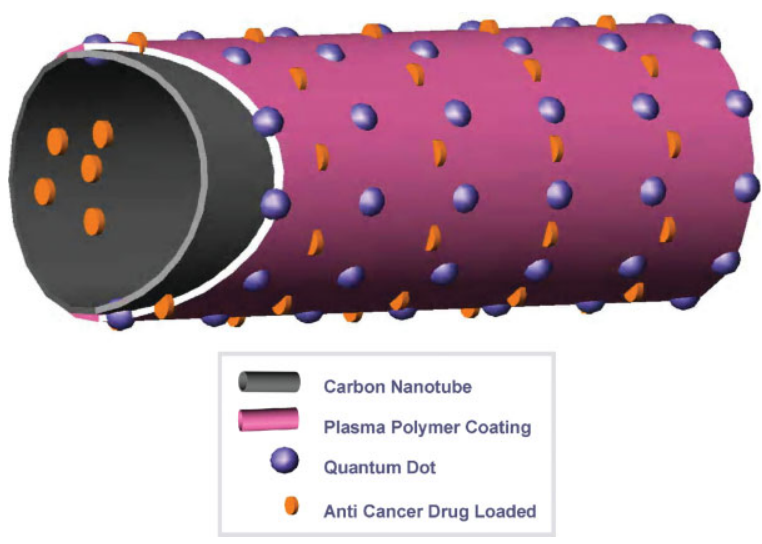

Figure 1. Schematic diagram illustrating the concept of a CNT functionalized with plasma polymer coating, luminescent QD, and loaded with anticancer drugs. The functionalized CNT can be used as biomarkers and drug carriers.

The experimental procedures and conditions for plasma surface functionalization and characterization have been published pre-viously. ${ }^{[4-7]}$ The plasma reactor for surface functionalization consisted mainly of a radiofrequency source, a glass vacuum chamber, and a press gauge. Monomers were introduced from the gas inlet during the plasma polymerization. In this study, acrylic acid (AA), lactic acid (LA), and glycolic acid (GA) were used as monomers for nanotube surface functionalization.

Figure $2 \mathrm{a}$ and $\mathrm{b}$ show high-resolution transmission electron microscopy (HRTEM) images of surface-functionalized CNT. An ultrathin AA film of $\sim 3 \mathrm{~nm}$ is clearly evident on the outer surface of the nanotube after plasma polymerization

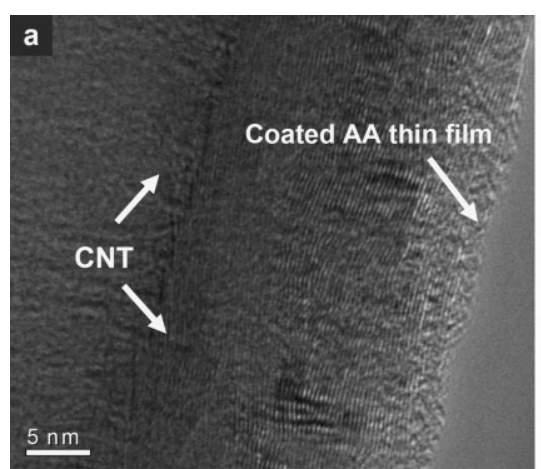

C
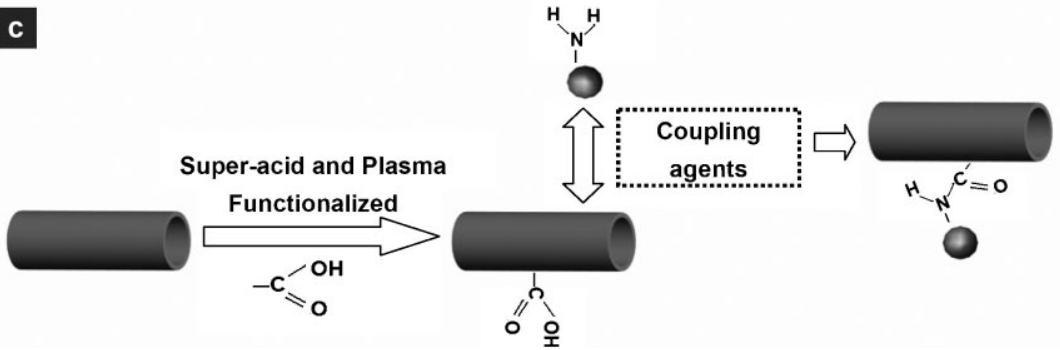

Figure 2. a) HRTEM image showing plasma deposited AA polymer thin film $(\sim 3 \mathrm{~nm})$ near the open end of the CNT, b) HRTEM image of the coating layer of PLCA on CNT by plasma polymerization, c) schematic diagram outlining chemical coupling procedure of amine-containing QD to carboxylfunctionalized CNT.
(Figure 2a). The characteristic lattice fringe spacing for carbon can be seen in contrast to the amorphous AA film (e.g., the absence of lattice fringes). The AA film appears quite uniform, covering the outer surface of the nanotube. Figure $2 b$ shows a HRTEM image of a PLGA-coating layer deposited on the CNT by plasma polymerization. LA and GA were used as monomers for this process. In this image, the thickness of ultrathin coating layer is approximately $6-7 \mathrm{~nm}$ and also appears as a uniform layer on the nanotube surface. For comparison, the inner diameter of CNT used is in the order of $50-80 \mathrm{~nm}$. This dimension appears particularly suitable for drug loading with anticancer drugs of comparable molecular size like paclitaxel. ${ }^{[26]}$ The polymer films deposited on nanotubes were characterized by surface analyses such as time of flight secondary ion mass spectroscopy (TOFSIMS), and results are detailed in ref. ${ }^{[4-7]}$.

\subsection{CNT-QD Conjugation}

The procedure for coupling amine-containing QD to AAfunctionalized CNT is illustrated in Figure 2c. Covalent attachment of QD to the CNT was achieved in the presence of 1-(3-dimethylaminopropyl)-3-ethylcarbodiimide hydrochloride (EDC) and $N$-hydroxysuccinimide (NHS). Conjugation of QD with nanotubes was confirmed by characterizing the microstructure of CNT-QD using HRTEM and energy dispersive spectroscopy (EDS), respectively. The TEM samples of CNT-QD were prepared by dispersing the solution directly onto holy carbon films supported with $\mathrm{Cu}$ grids. The TEM images of CNT-QD are shown in Figure 3. As can be seen in Figure. 3a, the QD exhibits a dark contrast with an average particle size of $5-10 \mathrm{~nm}$, randomly distributed on the surface of the nanotubes. In a $Z$-contrast TEM image of Figure $3 \mathrm{~b}$, these QD appears to be bright on the CNT surfaces with some aggregated clusters. QD coverage is not uniform throughout the entire length of the CNT suggesting preferential conjugation of $\mathrm{QD}$ due to inhomogeneous CNT dispersions.

Figure $3 \mathrm{c}$ and $\mathrm{d}$ show the HRTEM images of surface-conjugated QD. As can be seen in Figure 3c, the QD are quite distinct from the matrix of the CNT. The lattice images of QD and CNT are shown in Figure 3d. In this figure, the crystalline features of $\mathrm{CdSe} / \mathrm{ZnS}$ QD deposited on the surfaces of the CNTs can be clearly seen. The interlayers of the CNTs are also well resolved in both HRTEM images (Figure $3 \mathrm{c}$ and $\mathrm{d}$ ). These results suggest that the QD are either embedded within or attached onto the surface of the CNTs. 

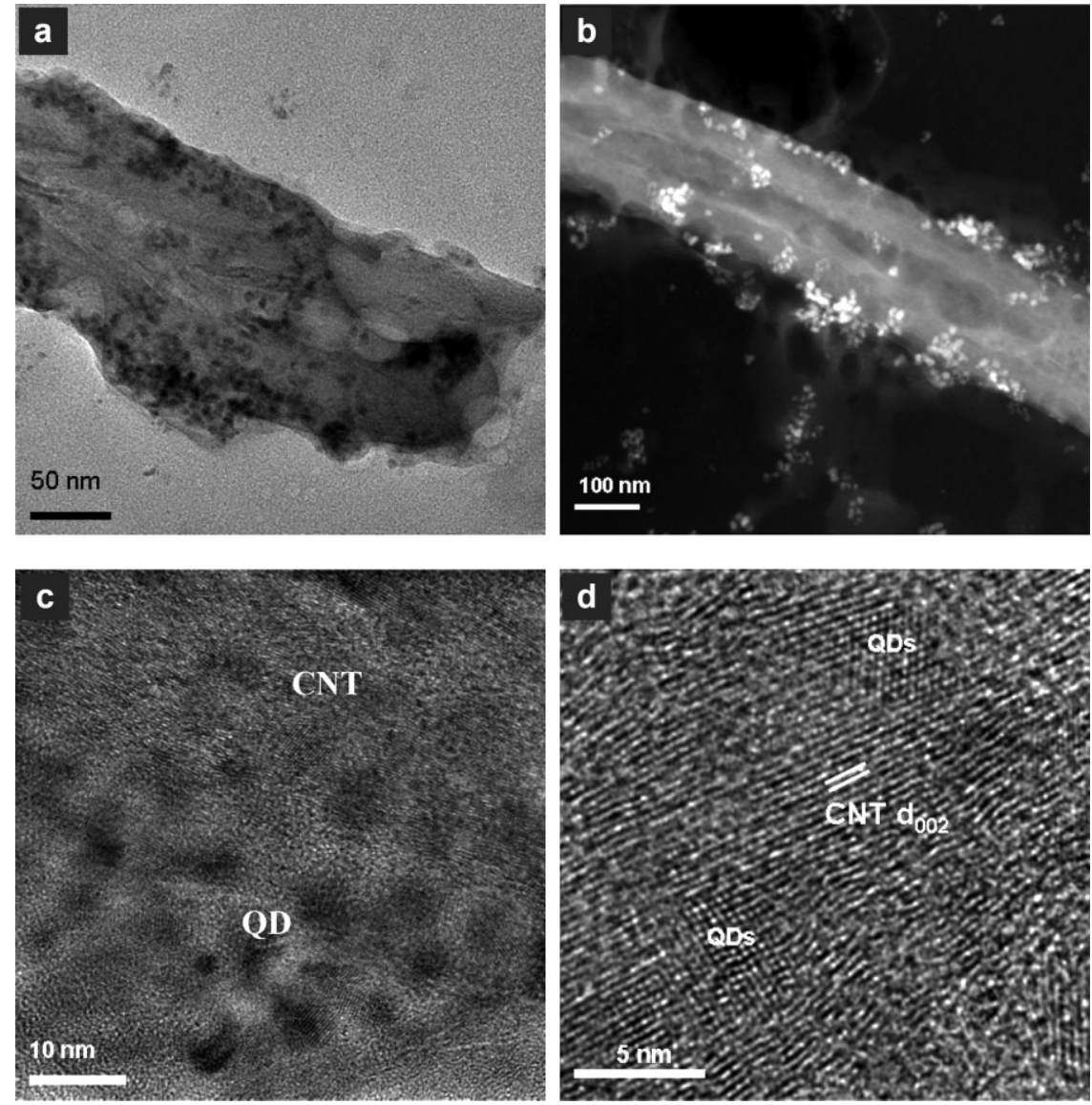

Figure 3. a) TEM image of CNT with surface coupled QD, b) Z-contrast TEM image showing the light gray QD on the CNT's surfaces, c) and d) HRTEM images of the crystalline CdSeTe/ZnS QD deposited on the surfaces of CNT.

Energy dispersive X-ray spectroscopy (EDS) measurements were performed by focusing the nanosized electron probe on CNT and QD as shown in Figure 4. Strong signals of Cd, $\mathrm{Se}, \mathrm{Te}, \mathrm{Zn}$, and $\mathrm{S}$ were observed in the spectrum acquired. These signals suggest the identity of the QD coupled onto the outer walls of CNT. The $\mathrm{Cu}$ and $\mathrm{C}$ peaks observed in the spectrum can be attributed to the TEM grids and CNT substrate. The EDS results confirm the presence of CdSe/ $\mathrm{ZnS}$ QD coupled onto the surface of the CNT. This is also consistent with the results of the HRTEM (Figure $3 c$ and d) showing the crystalline QD on the outside surface of a CNT.

It should be noted that no efficient coupling was observed when QD were simply mixed with $\mathrm{CNT}$ in various organic solvents (methanol, chloroform, and toluene) and in the absence of the activating agents. There was no obvious sign of QD degradation as studied by TEM (Figure 3). This enhanced chemical stability is consistent with the formation of an amide bond after covalent coupling between the amino group on the QD and the carboxyl group on the polymer-coated CNT. Furthermore, the preserved fluorescence of QD-modified CNT in vivo implies limited hydrolysis of this covalent coupling product by competent enzyme families such as peptideases.
Figure 5 compares the fluorescent spectra of uncoupled QD and the CNT-QD coupling product. As seen in this figure, the maximum emission of the uncoupled QD (dash dot line) is at $795.6 \mathrm{~nm}$, which is the consistent with the specification of this commercial product. For CNT-conjugated QD, the maximum emission (solid line) was shifted to $752.5 \mathrm{~nm}$, with a broad shoulder around $650 \mathrm{~nm}$. This shifting is likely attributable to the background emissions from the CNT or interactions between the QD and CNT. However, an understanding of the detailed physics of these interactions will require further investigation.

\subsection{Drug Storage and Cytotoxicity Study}

Paclitaxel-loaded CNTs were prepared by simple mixing of PLGA-coated CNT with a methanolic drug solution followed by evaporation of the organic solvent and repeated washes in distilled water. Drug loading efficiency was quantified by HPLC. The recovered amount of this anticancer agent from three independent production batches was $112.5 \pm 5.8 \mu \mathrm{g}$ $\mathrm{mg}^{-1}$ CNT. Variation in incubation time between drug solution and PLGA-coated CNT from 2 to $6 \mathrm{~h}$ did not significantly alter the relative loading efficiency (data not shown). Previously, paclitaxel encapsulation into polymeric microparticles was reported at loading capacities between 14 and $24 \mu \mathrm{g}$ drug $\mathrm{mg}^{-1}$ carrier material. ${ }^{[2,43]}$ Our results demonstrate a $\sim$ five-fold increase in this loading capacity when the polymer is deposited as a thin layer onto CNT using plasma polymerization. The reproducible drug

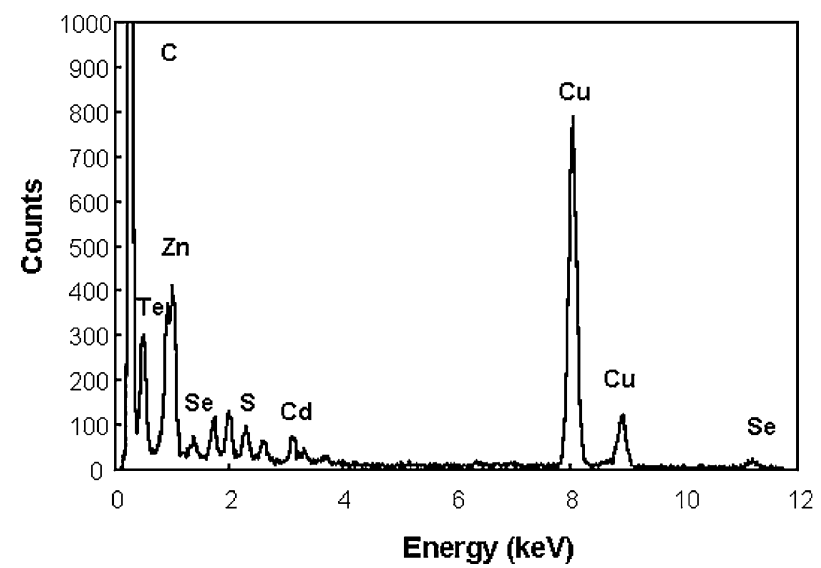

Figure 4. EDS acquired from QD attached on CNT's surface. It shows the elemental signals from the CdSeTe/ZnS QD and CNTs. 


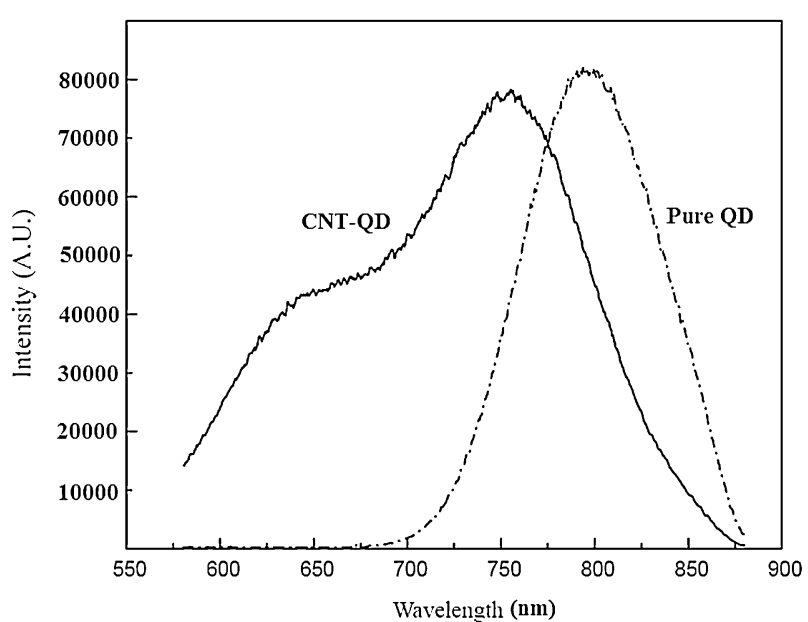

Figure 5. The fluorescent emission spectra of the uncoupled QD and CNT. QD. The maximum peak at $795.6 \mathrm{~nm}$ is consistent with specifications of this commercial QD. The blue shift from CNT-QD and a broad shoulder around $650 \mathrm{~nm}$ may result from the interactions and/or background emissions of CNT.

amount recovered after one single coating/drug loading cycle implies that drug load could be further augmented by several sequential polymer coating/drug loading steps. This is consistent with previous reports that identified high drug loading capacity of soluble CNT for the anticancer drug doxorubicin. ${ }^{[4]}$ Selection of methanol as solvent in our drug loading protocol was based on its high solubility capacity for paclitaxel and corresponding low solubility capacity for PLGA. As a consequence, we hypothesize that the PLGA film was not affected by the drug loading process and that paclitaxel is deposited by non-covalent adsorption either inside and/or outside of the PLGA-coated CNT. It is conceivable that conventional paclitaxel PLGA nanoparticles pre-fabricated using the solvent evaporation technique ${ }^{[45]}$ also adsorb to the surface of uncoated or coated CNT. Although this could constitute an alternative paclitaxel delivery system, reproducibility, and control of size distribution following adsorption of preformed nanoparticles are considered significant technical challenges and appear inferior to the simple drug loading procedure of PLGA-coated CNT described in this work.

The therapeutic efficacy of paclitaxel-loaded CNT was estimated in vitro by quantifying drug-induced changes in viability of human PC-3MM2 prostate cancer cells using the MTT assay. As shown in Figure 6, exposure of PC-3 human prostate cancer cells to paclitaxel for $96 \mathrm{~h}$ produced a dosedependent inhibition of mitochondrial dehydrogenase enzymes, which are essential for cell viability. The estimated paclitaxel concentration leading to a $50 \%$ inhibition of cell viability is $\sim 5 \mathrm{ng} \mathrm{mL}{ }^{-1}$ underlining the potency of this anticancer agent. When exposed to paclitaxel that was loaded onto PLGA-coated CNT, doses of $100 \mathrm{ng} \mathrm{mL}^{-1}$ of this novel drug delivery system are required to achieve an equivalent cell kill of $50 \%$. Importantly, viability of these human prostate cancer cells in the presence of PLGA-coated CNTs that did not contain paclitaxel was compromised only at doses
$>1000 \mathrm{ng} \mathrm{mL}^{-1}$. Whether coating with the biocompatible PLGA polymer truly altered the cytotoxic potential of CNTs or affected binding capacity of CNTs for the enzymatically produced formazan dye that is used to quantify cytotoxicity in this assay is unclear and will be subject of further investigations. ${ }^{[45,46]}$ However, it appears reasonable to conclude that the cytotoxic effect observed with drug-loaded CNTs in this in vitro model reflects the action of paclitaxel released from PLGA-coated carriers. Using the experimentally determined paclitaxel concentration that kills $50 \%$ of the tumor cells in the absence of CNTs (i.e., $5 \mathrm{ng} \mathrm{mL}^{-1}$ ) and the volume of $100 \mu \mathrm{L}$ media added per well, we can estimate a paclitaxel dose of $0.5 \mathrm{ng}$ released from the coated CNTs within $96 \mathrm{~h}$. Since the average drug load is $112.5 \mu \mathrm{g} \mathrm{mg}^{-1} \mathrm{CNT}$, the estimated $0.5 \mathrm{ng}$ paclitaxel corresponds to almost $50 \%$ of the total amount of anticancer agent incubated with the cells at the CNT dilution of $100 \mathrm{ng} \mathrm{mL}^{-1}$. To verify this prediction, more extensive drug release studies are required using different media in combination with cytotoxicity assessment.

\subsection{In vivo Imaging and Distribution of CNT-QD}

CNT-QD were injected i.v. via tail vein into mice and the emission at $800 \mathrm{~nm}$ was monitored in live animals at various time points (i.e., immediately after injection, 1, 2, 4, and 6 days) using the Kodak 4000MM whole mouse imaging system. The in vivo images were acquired from the front, side, and back of the animal using the whole body mode. Mice are known to have visible emissions that may overlap with those from the $\mathrm{QD}$, especially near $600 \mathrm{~nm}$. After subcutaneous injection in mice, the CNT-coupled QD exhibited luminescent emissions in the visible light range. QD with longer wavelengths, however, provide the basis for much brighter imaging, which is suitable for deep tissue diagnosis, due to reduced background. In this

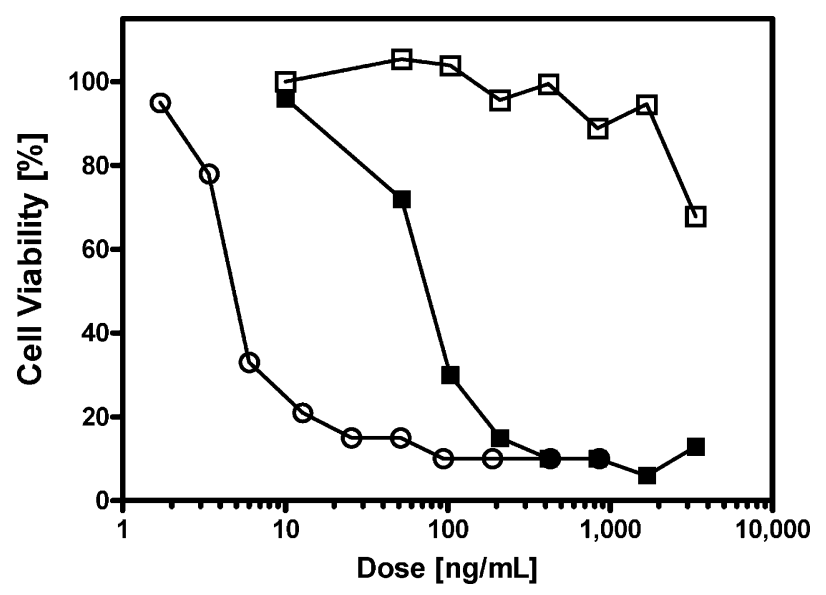

Figure 6. Dose-dependent effects of paclitaxel and various CNTs on viability of human PC-3MM2 prostate cancer cells. Tumor cells were treated for 4 days with various concentrations of paclitaxel $(O)$, paclitaxel-loaded, PLGA-coated CNTs $(\square)$, or PLGA-coated CNTs without drug $(\square)$ either dissolved or dispersed in culture media supplemented with $3 \%$ FBS for $96 \mathrm{~h}$. Each data point represents the mean of six replicate experiments. 


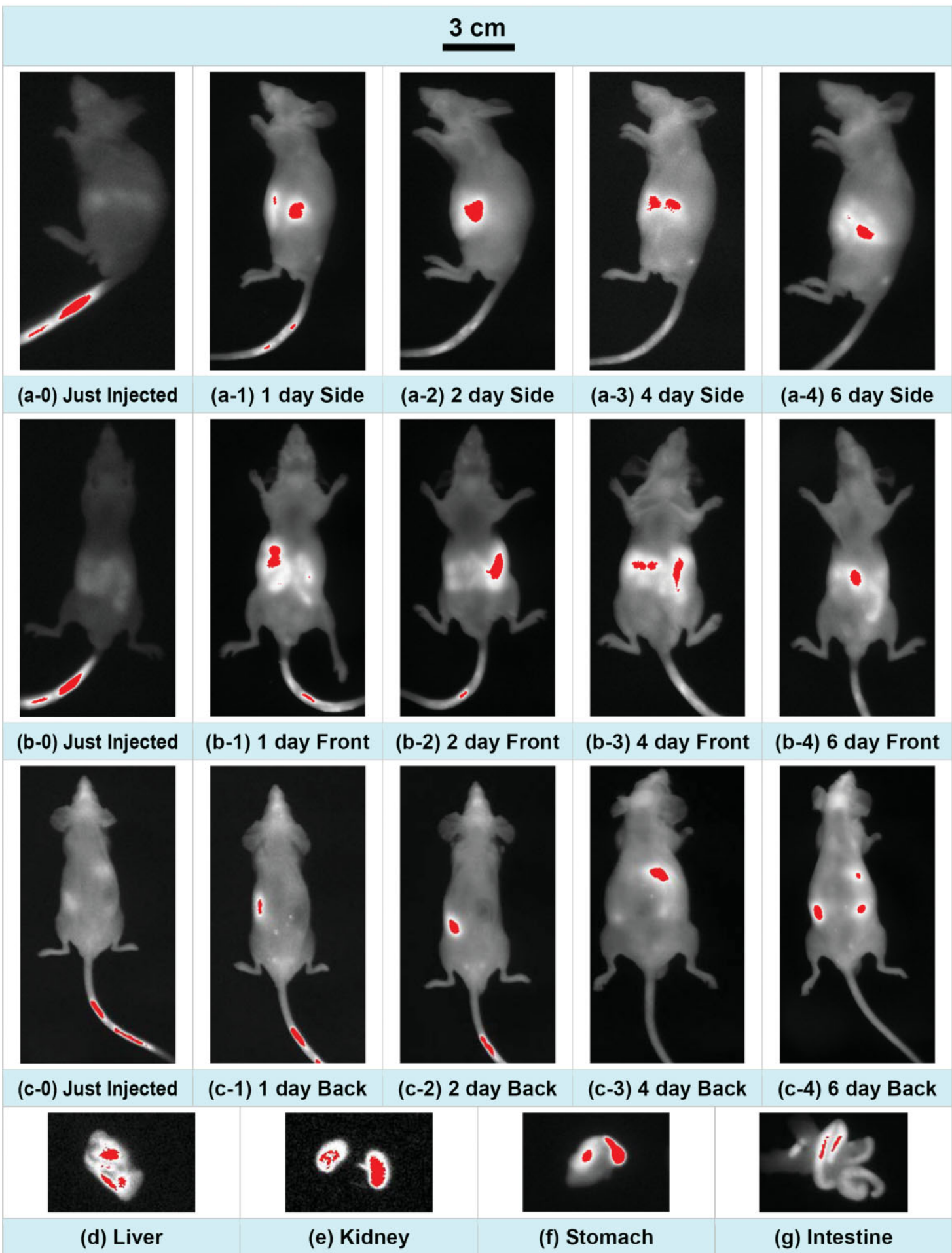

Figure 7. In vivo fluorescence images of CNT-QD IV injected into nude mice and imaged after circulation at various time intervals; (a-0 to a-4) the images taken from the side of mice; (b-0 to b-4) from the front of mice; (c-0 to c-4) from the back of mice, and d), e), f), g) organ images taken after having sacrificed the mice on the 6th day of post-injection. These pictures show prominent CNT-QD uptaking in the liver, kidney, stomach, and intestine.

experiment, the QD with emission of $800 \mathrm{~nm}$ was shown to be capable of exhibiting much brighter images as compared with those with emissions at $600 \mathrm{~nm}$ at the same depth. The small size of CNT-QD conjugates should allow efficient tissue perfusion and good targeting to specific sites in the tissues, provided that the QDs remain in circulation for a long enough time. Figure 7 shows spectral imaging results obtained from CNT-QD i.v. injected into the tail vein of mice. 
Due to high quantum yields and absorbency, the fluorescence of CNT-QD in the superficial vasculature (tail veins in Figure 7 a-1, b-1, c-1) was readily visible under the Kodak Imaging System immediately after injection. However, at this early post-injection stage, the CNT-QD cannot be imaged in organs and tissues. After circulation for 2-6 days, the images (Figure 7 a-2 to a-4, b-2 to b-4, c-2 to c-4) exhibit strong signals of CNT-QD in several organs of live animal including liver, kidney, stomach, and intestine. The enhanced contrast in these images indicates that CNT-QD can be visualized against an essentially black background, with little or no interference by auto-fluorescence of the animal. The results indicate that QD with emission at $800 \mathrm{~nm}$ are well suited for fluorescence visualization in live animals. To confirm CNT-QD uptake in these organs, mice were sacrificed and liver, kidney, stomach, and intestine were harvested for ex vivo imaging (Figure $7 \mathrm{~d}-\mathrm{g}$ ). The strong luminescent emissions in these organs under epiUV illumination support our conclusions that CNT-QD may provide significant advantages for deep tissue diagnosis.

It must be emphasized that the tested CNT did not contain specific cell or organ targeting moieties. Therefore, the observed biodistribution pattern following i.v. administration reflects the results of non-specific binding and elimination processes. It is expected, however, that coupling with specific targeting ligands such as peptides and antibodies will alter the biodistribution pattern.

After i.v. injection of CNT-QD, liver, kidney, stomach, and intestine samples were collected for a period of 6 days and analyzed by inductively coupled plasma mass spectrometry (ICP-MS). The results of this quantitative evaluation are presented in Figure 8. In general, CNT-QD accumulated to the greatest extent in the liver. One day after injection, the mass of $\mathrm{Cd}$ is $70.36 \mu \mathrm{g} \mathrm{g}^{-1}$ in liver; $2.42 \mu \mathrm{g} \mathrm{g}^{-1}$ in kidney; $0.86 \mu \mathrm{g} \mathrm{g}^{-1}$ in stomach, and $1.00 \mu \mathrm{g} \mathrm{g}^{-1}$ in intestine, respectively. After 3 days, CNT-QD associated with liver, stomach, and intestine significantly decreased, whereas kidney levels appeared

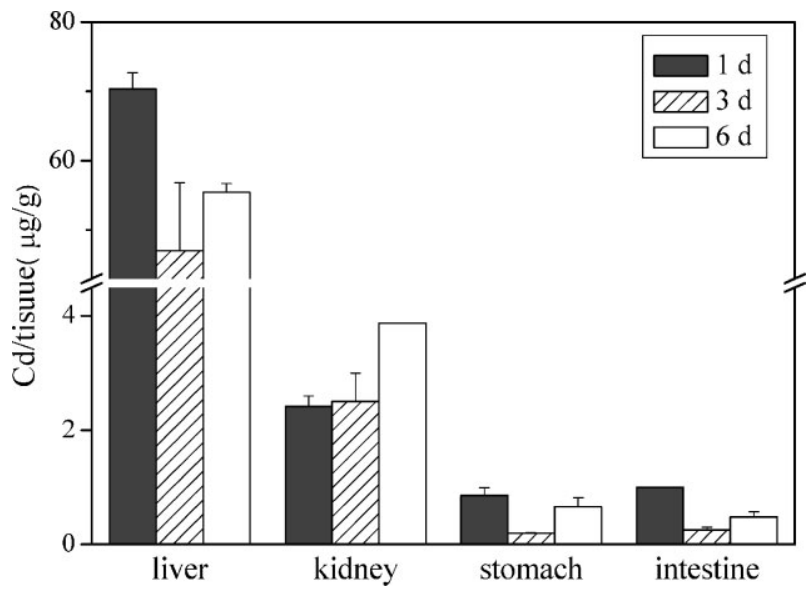

Figure 8. In vivo distribution of CNT coupled with $\mathrm{CdSeTe} / \mathrm{ZnS}$ after IV injected in the nude mice for 1, 3, and 6 days. The liver, kidney, stomach, and intestine of the mice were collected, respectively. The digested organ samples were analyzed for $\mathrm{Cd}$ mass by using an ICP-MS. unchanged. At the sixth day, quantified $\mathrm{Cd}$ amounts slightly increased in all organs without dramatic changes in the relative distribution (i.e., liver $>$ kidney $>$ stomach $\sim$ intestine). These data confirm results from whole body in vivo imaging acquired in live animals as shown in Figure 7.

\section{Conclusions}

Based on a novel nanostructure design, QD were conjugated onto the surfaces of the CNT for in vivo imaging. Anticancer drug was efficiently loaded in PLGA-coated CNT. Plasma surface modification of CNT with acidic polymers effectively facilitated coupling of amine-containing QD. The CNT-QD formed a fluorescent domain of emission in the desired wavelength range. With a near infrared emission around $800 \mathrm{~nm}$, the CNT-QD exhibited strong luminescence suitable for non-invasive optical in vivo imaging. The PLGA-coated CNT showed high loading efficiency for paclitaxel, which demonstrated in vitro antitumor efficacy against human PC3MM2 prostate cancer cells. ICP-MS studies indicated predominant CNT-QD uptake in liver, kidney, stomach, and intestine following i.v. administration in mice. The development of CNT-QD as a non-invasive optical in vivo imaging and drug delivery system may have a great impact in early detection, diagnosis, and treatment of cancer.

\section{Experimental}

CNT-QD Conjugation: Commercial grade multiwall CNTs were obtained from Applied Science, Inc. The CNT were chosen for their relatively larger inner wall diameters $(50-80 \mathrm{~nm})$, which offer increased capacity for drug storage. The as-produced CNT were severely aggregated and highly hydrophobic. To modify surface properties, CNTs were initially treated with superacid $\left(\mathrm{HNO}_{3}+\mathrm{H}_{2} \mathrm{SO}_{4}\right)$. Subsequently, an ultra-thin AA polymer film was deposited on these superacid-treated CNT by plasma coating using a protocol published previously by our laboratories ${ }^{[4-7]}$. Briefly, the input power was set to $40 \mathrm{~W}$, and the system pressure was adjusted from 150 to 400 mTorr. Plasma treatment time was $30 \mathrm{~min}$ per batch. After surface functionalization, the CNT were dispersed in phosphate-buffered saline solution (PBS) and sonicated for $30 \mathrm{~min}$. The as-synthesized CNTs were too long for in vivo circulation. To select CNTs in a more desirable size range around $200-400 \mathrm{~nm}$, a sequential filtration protocol was applied using $25 \mathrm{~mm}$ nylon syringe filters with pore sizes of 0.45 and $0.22 \mu \mathrm{m}$, respectively (Thermo Fisher Scientific, Inc., Pittsburgh, PA). A stable suspension of CNT collected from the $0.22 \mu \mathrm{m}$ filter in PBS was used for i.v. administration in mice.

Amine-functionalized Qdot 800 ITK QD with emission wavelength of $800 \mathrm{~nm}$ were supplied by Invitrogen Corporation (Carlsbad, CA Product \# Q21571MP, $8 \mu$ M solution). QD have a core of CdSeTe and a shell of $\mathrm{ZnS}$ with a surface coating of amino PEG. Coupling of the amine-containing QD to the carboxyl-functionalized CNT was achieved using standard carbodiimide chemistry ${ }^{[47}$ and the procedure is illustrated in Figure 2c. Briefly, $10 \mu \mathrm{g}$ of CNT were dispersed into $100 \mu \mathrm{L}$ PBS and combined with $200 \mu \mathrm{L}$ of a solution containing $0.5 \mathrm{M}$ EDC and $1 \mathrm{M}$ NHS in PBS. Following a $30 \mathrm{~min}$ activation at room temperature $20 \mu \mathrm{L}(0.16 \mathrm{nmol})$ of the commercial, aminofunctionalized QD suspension in borate buffer, $\mathrm{pH} 8.3$ were added, and the CNT-QD coupling mixture was incubated at $50{ }^{\circ} \mathrm{C}$ for $12 \mathrm{~h}$. The suspension was then cooled to room temperature, centrifuged at 
$12000 \mathrm{rpm}$ for $3 \mathrm{~min}$, and rinsed three times with PBS using centrifugation and decantation. The final CNT-QD preparation was re-suspended into $200 \mu \mathrm{L}$ of PBS.

Luminescence Spectroscopy: In order to obtain the emission spectrum of the sample, $475 \mathrm{~nm}$ LED was used as the exciting source. A $550 \mathrm{~nm}$ long path filter was placed into the system to block the emissions from the diode. The fluorescence emission was dispersed by a SPEX 1704 monochromator and detected with a cooled RCA C31034 photomultiplier. An electric motor driving chopper combined with SR830 DSP lock-in amplifier was used to improve the signal/noise ratio of the data. Both measurements were performed at room temperature and recorded using our own software.

Paclitaxel Storage in PLGA-coated CNTs and Cytotoxicity Study: PLGA-coated CNT were prepared by plasma polymerization and washed twice with methanol. Drug loading was performed by incubating $20-25 \mathrm{mg}$ of PLGA-coated CNT with $11.4-14.2 \mathrm{~mL}$ of a $0.7 \mathrm{mg} \mathrm{mL}^{-1}$ paclitaxel stock solution prepared in methanol at a final $\mathrm{CNT} /$ drug ratio of $2.5 \mathrm{w} / \mathrm{w}$. The suspension was sonicating with an energy output of $5 \mathrm{~W}$ in a pulse mode for 5 min under cooling and vacuum dried. Removal of the organic solvent under these conditions was completed within $2 \mathrm{~h}$. Preparations were washed at least three times with $10 \mathrm{~mL}$ of distilled water followed by centrifugation. After the last wash, paclitaxel-loaded CNT suspended in water were frozen and lyophilized until further use. The amount of paclitaxel loaded onto PLGA-coated CNT was quantified by HPLC after back extraction of the drug from CNT using methanol. Drug-loaded CNT $(1.5-2 \mathrm{mg})$ was suspended in $5 \mathrm{~mL}$ of methanol, sonicated for $10 \mathrm{~min}$ under cooling, and centrifuged. The supernatant organic phase was removed, and the procedure was repeated twice. An aliquot of the pooled extraction solutions was evaporate to dryness under $\mathrm{N}_{2}$ and reconstituted in acetonitrile. Samples $(10 \mu \mathrm{L})$ were injected into a Varian ProStar HPLC system equipped with an UV detector. Isocratic chromatography was performed on a Dynamax C18 column using acetonitrile/ water $(60: 40)$ as a mobile phase. Paclitaxel was detected at $\lambda=220 \mathrm{~nm}$ and quantified using a standard curve between 0 and $250 \mu \mathrm{g} \mathrm{mL}{ }^{-1}$. Drug loading was expressed as amount of drug per weight of carrier material (i.e., $\mu \mathrm{g}$ paclitaxel $\mathrm{mg}^{-1} \mathrm{CNT}$ ). Mass balance of this drug loading and backextraction protocol was verified by separate incubation of fabrication containers and drug-loaded CNT with acetonitrile that exhibit high solubility capacity for both paclitaxel and PLGA. Quantitative evaluation of these samples revealed $20-30 \%$ drug loss in fabrication containers and $>90 \%$ recovery of drug after the described backextraction protocol.

PC-3MM2 human prostate cancer cells were cultured in Eagle's minimal essential medium (EMEM) supplemented with 5\% FBS, nonessential amino acids, sodium pyruvate, vitamin A, and glutamine at $37^{\circ} \mathrm{C}$ in a humidified incubator under an atmosphere of $5 \% \mathrm{CO}_{2}$. Cells in exponential growth phase were harvested by a 2 min treatment with a $0.25 \% \operatorname{trypsin} / 0.02 \%$ EDTA solution and maintained by periodic dilutions with a fresh medium. Effects of paclitaxel solutions and paclitaxel-loaded CNT suspensions on in vitro cell viability were evaluated using the MTT [3-(4,5-dimethylthiazol-2-yl)-2,5-diphenyltetrazolium bromide] assay. Tumor cells were plated in $100 \mu \mathrm{L}$ EMEM- $1 \%$ FBS at a density of 1000 cells in a 96-well plate. After an overnight incubation culture period, cells were exposed for 4 days with different concentrations of paclitaxel or different amounts of paclitaxel-loaded $\mathrm{CNT}$ at $37{ }^{\circ} \mathrm{C}$ under standard cell culture conditions. EMEM- $1 \%$ FBS was used as vehicle control. 3-(4,5-Dimethylthiazol-2-yl)-2,5-diphenyltetrazolium bromide $\left(2 \mathrm{mg} \mathrm{mL}^{-1}\right.$ in PBS) was added to the cultures at $0.05 \mathrm{~mL}$ per well during the final $2 \mathrm{~h}$ of incubation. The medium was then carefully removed, and the dark blue formazan was dissolved in $100 \mu \mathrm{L}$ per well of DMSO. The absorbance of each well was measured with a FluoStar Optima multidetection microplate reader (BMG Labtechnologies, Durham, NC) at $570 \mathrm{~nm}$. The percentages cell viability were calculated according to: (Abs $570 \mathrm{~nm}$ of treated group/ Abs $570 \mathrm{~nm}$ of control group) 100 .

In Vivo Imaging of CNT-QD: Whole body images were acquired using a Kodak4000 MM Whole-Mouse Image Station. Excitation and emission filters were 720 and $790 \mathrm{~nm}$, respectively, centered on the emission maxima of the QD. The excitation light was from an UV lamp with the $10 \times$ zoom lens, and the exposure time was $2 \mathrm{~min}$. The dosage used in this study was $5 \mu \mathrm{g}$ of CNT coupled with $0.08 \mathrm{nmol}$ of QD and dispersed in $100 \mu \mathrm{L}$ PBS. $N u / n u$ nude mice, obtained from the National Cancer Institute, MD, were 6-8 weeks old and typically weigh $18 \mathrm{~g}$. Mice were anesthetized for imaging by beutal saline intraperitoneally. The mice used for in vivo imaging were maintained in a facility approved by the American Association for Accreditation of Laboratory Animal Care and in accordance with current regulations and standards of the US Department of Agriculture, US Department of Health and Human Services and National Institute of Health. This study was approved by Institutional Animal Use and Care Committee (IACUC) at the University of Cincinnati (OH).

Distribution of CNT-QD In Vivo: After UV irradiation for $30 \mathrm{~min}$, $100 \mu \mathrm{L}$ of CNT-QD ( $5 \mu \mathrm{g}$ of CNT coupled with $0.08 \mathrm{nmol}$ of QD) were injected i.v. into nude mice via the tail vein. The liver, kidney, stomach, and intestine of the mice were collected for 6 days and analyzed by ICPMS. Each organ sample was washed thoroughly with deionized water and dried for $4 \mathrm{~h}$ at $120^{\circ} \mathrm{C}$. Each experiment was repeated three times. In order to prepare ICP-MS solution, the organ samples were digested by a microwave accelerated reaction system (CEM MARS 240/50), following standard protocols. The digested solutions were then qualitatively analyzed for the mass of Cd by using an ICP-MS (Perkin Elmer, SCIEX ELAN DRC-e). Each sample was analyzed five times.

Received: March 21, 2008 Revised: May 21, 2008 Published online: August 8, 2008

[1] K. Balasubramanian, M. Burghard, Small 2005, 1, 180

[2] J. Liu, A. G. Rinzler, H. Dai, J. H. Hafner, R. K. Bradley, P. J. Boul, A. Lu, T. Iverson, K. Shelimov, C. B. Huffman, F. Rodriguez-Macias, Y. Shon, T. R. Lee, D. T. Colbert, R. E. Smalley, Science 1998, 280, 1253.

[3] S. Banerjee, S. S. Wong, Nano Lett. 2002, 2, 195.

[4] D. Shi, P. He, J. Lian, L. M. Wang, D. Mast, M. Schulz, Appl. Phys. Lett. 2002, 81, 5216

[5] D. Shi, S. X. Wang, W. J. van Ooij, L. M. Wang, J. G. Zhao, Z. Yu, Appl. Phys. Lett. 2001, 78, 1243.

[6] D. Shi, P. He, J. Lian, L. M. Wang, D. Mast, M. Schulz, Appl. Phys. Lett. 2003, 83, 5301.

[7] D. Shi, P. He, J. Lian, L. M. Wang, W. J. Van Ooij, J. Mater. Res. 2002, 17, 981.

[8] M. J. O'Connell, S. M. Bachilo, C. B. Huffman, V. Moore, M. S. Strano, E. Haroz, K. Rialon, P. J. Boul, W. H. Noon, C. Kittrell, J. Ma, R. H. Hauge, R. B. Weisman, R. E. Smalley, Science 2002, 297, 593.

[9] S. M. Bachilo, M. S. Strano, C. Kittrell, R. H. Hauge, R. E. Smalley, R. B. Weisman, Science 2002, 298, 2361.

[10] P. W. Barone, S. Baik, D. A. Heller, M. S. Strano, Nat. Mater. 2004, 4, 86.

[11] S. Wray, M. Cope, D. T. Delpy, Biochim. Biophys. Acta 1988, 933, 184.

[12] L. J. McCartney, J. C. Pickup, O. J. Rolinski, D. J. S. Birch, Anal. Biochem. 2001, 292, 216.

[13] N. Klonis, N. H. Quazi, L. W. Deady, A. B. Hughes, L. Tilley, Anal. Biochem. 2003, 317, 47.

[14] S. Kim, Y. T. Lim, E. G. Soltesz, A. M. De Grand, J. Lee, A. Nakayama, J. A. Parker, T. Mihaljevic, R. G. Laurence, D. M. Dor, L. H. Cohn, M. G. Bawendi, J. V. Frangioni, Nat. Biotechnol. 2004, 22, 93

[15] J. V. Frangioni, Curr. Opin. Chem. Biol. 2003, 7, 626.

[16] D. Shi, J. Lian, W. Wang, G. K. Liu, P. He, Z. Dong, L. M. Wang, R. C. Ewing, Adv. Mater. 2006, 18, 189

[17] L. Brus, Appl. Phys. A 1991, 53, 465.

[18] A. P. Alivisatos, J. Phys. Chem. 1996, 100, 13226. 
[19] S. Banerjee, S. S. Wong, Nano Lett. 2002, 2, 195.

[20] J. M. Haremza, M. A. Hahn, T. D. Krauss, Nano Lett. 2002, 2, 1253.

[21] W. C. W. Chan, S. M. Nie, Science 1998, 281, 2016.

[22] M. Bruchez, Jr., M. Moronne, P. Gin, S. Weiss, A. P. Alivisatos, Science 1998, 281, 2013.

[23] W. C. Chan, S. Nie, Science 1998, 281, 2016.

[24] S. J. Rosenthal, I. Tomlinson, E. M. Adkins, S. Schroeter, S. Adams, L. Swafford, J. McBride, Y. Wang, L. J. DeFelice, R. D. Blakely, J. Am. Chem. Soc. 2002, 124, 4586.

[25] B. Dubertret, P. Skourides, D. J. Norris, V. Noireaux, A. H. Brivanlou, A. Libchaber, Science 2002, 298, 1759.

[26] J. K. Jaiswal, H. Mattoussi, J. M. Mauro, S. M. Simon, Nat. Biotechnol. 2002, 21, 47.

[27] M. E. Akerman, W. C. W. Chan, P. Laakkonen, S. N. Bhatia, E. Ruoslahti, Proc. Natl. Acad. Sci. USA 2002, 99, 12617.

[28] I. Brigger, C. Dubernet, P. Couvreur, Adv. Drug Deliv. Rev. 2002, 54 , 631.

[29] B. Ballou, B. C. Lagerholm, L. A. Ernst, M. P. Bruchez, A. S. Waggoner, Bioconjugate Chem. 2004, 15, 79.

[30] G. Jia, H. Wang, L. Yan, X. Wang, R. Pei, T. Yan, Y. Zhao, X. Guo, Environ. Sci. Technol. 2005, 39, 1378.

[31] C. W. Lam, J. T. James, R. McCluskey, R. L. Hunter, Toxicol. Sci. 2004, 77, 126.

[32] A. M. Derfus, W. C. W. Chan, S. N. Bhatia, Nano Lett. 2004, 4, 11.

[33] B. Dubertret, P. Skourides, D. J. Norris, V. Noireaux, A. H. Brivanlou, A. Libchaber, Science 2002, 298, 1759.
[34] J. K. Jaiswal, H. Mattoussi, J. M. Mauro, S. M. Simon, Nat. Biotechnol. 2003, $21,47$.

[35] W. J. Parak, R. Boudreau, M. Le Gros, D. Gerion, D. Zanchet, C. M Micheel, S. C. Williams, A. P. Alivisatos, C. Larabell, Adv. Mater. 2002, 14,882

[36] B. Ballou, B. C. Lagerholm, L. A. Ernst, M. P. Bruchez, A. S. Waggoner, Bioconjugate Chem. 2004, 15, 79.

[37] D. R. Larson, W. R. Zipfel, R. M. Williams, S. W. Clark, M. P. Bruchez, F. W. Wise, W. W. Webb, Science 2003, 300, 1434.

[38] A. Hoshino, K. Hanaki, K. Suzuki, K. Yamamoto, Biochem. Biophys. Res. Commun. 2004, 314, 46.

[39] C. M. Spencer, D. Faulds, Drugs 1994, 48, 794.

[40] M. Ishitobi, E. Shin, N. Kikkawa, Int. J. Clin. Oncol. 2001, 6, 55.

[41] M. Schmitt-Sody, S. Strieth, S. Krasnici, B. Sauer, B. Schulze, M. Teifel, U. Michaelis, K. Naujoks, M. Dellian, Clin. Cancer Res. 2003, 9 , 2335.

[42] G. S. Das, G. H. R. Rao, R. F. Wilsion, T. Chandy, J. Biomed. Mater. Res. 2001, 55, 96.

[43] Y. Dong, S. S. Feng, Biomaterials 2005, 26, 6068.

[44] Z. Liu, X. Sun, N. Nakayama-Ratchford, H. Dai, ACS Nano 2007, 1, 50

[45] J. M. Wörle-Knirsch, K. Pulskamp, H. F. Krug, Nano Lett. 2006, 6 , 1261.

[46] M. Davoren, E. Herzog, A. Casey, B. Cottineau, G. Chambers, H. Byrne, F. M. Lyng, Toxicol. in Vitro 2007, 21, 438.

[47] G. T. Hermanson, Bioconjugate Techniques, Academic Press, London, UK 1996, p. 8. 